\title{
Pre-operative scoring system to determine the surgical strategy for periprosthetic hip infection
}

\author{
Kenichi Oe $\cdot$ Hirokazu Iida $\cdot$ Narumi Ueda $\cdot$ \\ Tomohisa Nakamura $\cdot$ Naofumi Okamoto $\cdot$ Yusuke Ueda
}

Received: 5 May 2014 / Accepted: 30 July 2014 / Published online: 17 August 2014

(C) The Author(s) 2014. This article is published with open access at Springerlink.com

\begin{abstract}
Purpose The aim of this study was to evaluate the results of one- and two-stage revision total hip arthroplasties (THAs) and to determine a rational surgical treatment strategy for periprosthetic hip infections.

Methods We constructed a 12-point preoperative scoring system to suggest either one- or two-stage revision THAs, based on a retrospective analysis of 55 operative procedures. Prosthesis survival was analysed using the Kaplan-Meier method, and the validity of the scoring system was evaluated using receiver-operating characteristic curves.

Results At the end point of implant removal due to recurrent infection, the ten year survival rates were 94 and $87 \%$ for oneand two-stage revision THAs, respectively. One-stage revision THA was recommended for patients scoring $>9$ points. The risk of recurrent infection in patients scoring 4 points was $83 \%$. The sensitivity and specificity of a cut-off value of 4 points, determined by the scoring system, were 83 and $100 \%$, respectively.

Conclusions The novel pre-operative scoring system was useful for the management of periprosthetic hip infections.
\end{abstract}

Keywords Infection · Total hip arthroplasty · One-stage revision $\cdot$ Two-stage revision $\cdot$ Scoring system

\section{Introduction}

Treatments for periprosthetic hip infections remain controversial and are particularly challenging because most patients require implant removal. In North America, the gold standard

K. Oe $(\triangle) \cdot$ H. Iida $\cdot$ N. Ueda $\cdot$ T. Nakamura $\cdot$ N. Okamoto $\cdot$

Y. Ueda

Department of Orthopaedic Surgery, Kansai Medical University,

2-5-1 Shinmachi, Hirakata-city, Osaka 573-1010, Japan

e-mail: oeken@hirakata.kmu.ac.jp treatment for periprosthetic hip infection is two-stage revision total hip arthroplasty (THA). In contrast, in Europe, one-stage revision THA, which includes replacement of the prosthesis during the same surgical procedure, is often preferred. This is likely because one-stage revision THA usually employs an antibiotic-loaded acrylic cement (ALAC) [1], and for over a decade, the North American trend has been towards the use of 'cementless' techniques. According to some meta-analyses, the infection control rate in two-stage revision THAs, using ALAC, was $88-93 \%$, whereas that for one-stage revision THAs, also with ALAC, was $82-86 \%$ [2-5]. On the other hand, without ALAC, infection control was achieved in $82-91 \%$ of two-stage revision THA cases and in 56-59\% of one-stage revision THAs [2-5]. The two-stage revision THA is safe, but has the disadvantages of requiring a second operation and having higher associated costs. Currently, there are algorithms and staging systems for periprosthetic hip infections [6-10], so it may be common for surgeons to employ one- or two-stage revision THA as the situation demands. However, a scoring system is not available to definitively determine the appropriate surgical strategy for patients with periprosthetic hip infections.

We performed one- and two-stage revision THAs for periprosthetic hip infection, according to various published criteria [6, 7, 9-12]. Here, we attempted to set up a scoring system for the pre-operative evaluation of one- and two-stage revision THAs, based on a retrospective analysis of 55 cases. The aim of this study was to report the scoring system and to determine a rational surgical treatment strategy. The hypothesis of the study was that a scoring system would facilitate better management of periprosthetic hip infections.

\section{Patients and methods}

Between February 2001 and December 2011, 68 consecutive revision THAs were performed at our institution for 
periprosthetic hip infections. One-stage revision THAs were performed on 21 hips; two-stage revision THAs were performed on 47 hips. The two-stage revision THAs included six multiple-stage revision THAs that required $\geq$ two debridements and a single implantation. Periprosthetic hip infection or healing was evaluated according to the criteria of Giulieri et al. [13]. Infection was diagnosed if a sinus tract was present and communicating with the joint space or if at least two of the following criteria were met: (1) a positive bacterial culture from at least one intra-operative tissue specimen or the joint aspirate, (2) the presence of neutrophils in the tissue specimens, (3) clinical and laboratory signs of infection or (4) radiological signs of infection. Healing was defined as the lack of clinical signs and symptoms of infection, a C-reactive protein (CRP) level $<10 \mathrm{mg} / 1$ or an erythrocyte sedimentation rate $<20 \mathrm{~mm} / \mathrm{h}$ and the absence of radiological signs of infection at the follow-up visit $>24$ months after the first revision. Therefore, a successful case was defined as the absence of infection at the $>24$-month, post-revision follow-up visit; failure was defined as implant removal due to recurrent infection. Of the total number of patients undergoing revision THAs, 13 returned to their original referring hospital and were lost to follow-up. Based on telephone consultations, the 13 patients did not undergo additional revisions, but were excluded from the analysis. In this study, therefore, a total of 55 hips were analysed (Fig. 1) in 43 women and 12 men. The patients had a mean age, at the time of surgery, of 66 (range 34-90) years. Infected implants were associated with THAs in 32 patients and hemiarthroplasties in 23 patients.

In accordance with published criteria [6, 7, 9-12], onestage revision THA was performed if (1) the patient's general condition was good, (2) wound complications were absent, (3) the pathogen(s) was antibiotic sensitive and (4) the bone defect requiring reconstruction was small. Two-stage revision THA was performed in cases that did not meet the criteria for one-stage revision THA, but the final decision was made intraoperatively by the surgeon [14]. All patients received cemented THAs with custom-mixed ALAC, according to the reported bacterial sensitivities determined from the preoperative aspirates. Over $3.6 \mathrm{~g}$ of antibiotic per $40 \mathrm{~g}$ of cement was used in the first stage of two-stage revision THAs, and $1.0-2.0 \mathrm{~g}$ of antibiotic per $40 \mathrm{~g}$ of cement was used in the second stage of two-stage revision THAs and in one-stage revision THAs (Endurance Bone Cement, DePuy International, Leeds, UK).

Treatment of periprosthetic hip infection involves thorough debridement and administration of adequate local and systemic antibiotics. Initially, a thorough debridement was performed, using a transgluteal approach, to effect the complete removal of all implant components, cement, granulation tissue and necrotic/infected tissues. To ensure a thorough debridement, routine radiographs, computed tomography scans and radioactive isotopes were used to assess necrotic and infected tissues. Aspiration and periprosthetic tissue specimens were sent for microbiology and pathology studies. The hip joint was exhaustively flushed and then irrigated with hydrogen peroxide solution and povidone iodine. Subsequently, for onestage revision THAs, all instruments, including the light handle, sucker and electrocautery scalpel, and protective clothing were replaced with fresh, sterile materials. Further, the old drape was covered with a new one, and new sterile instruments were prepared. Appropriate intravenous systemic antibiotic therapy was started and continued for two weeks postoperatively. Subsequently, oral antibiotic therapy, tailored to the clinical signs and CRP levels, was administered for a minimum of three months. In two-stage revision THAs, after thorough debridement, a handmade rod and beads, including adequate high-dose ALAC were temporarily placed. Appropriate intravenous systemic antibiotic therapy was initiated and continued postoperatively for two weeks, followed by oral antibiotic therapy during the period between the initial debridement and the revision THA. At six to eight weeks after the first thorough debridement, the clinical signs and CRP levels were reviewed and a second thorough debridement was performed prior to the THA; the THA, using ALAC, was then performed. The postoperative therapy was identical to that used following one-stage revision THAs. If the debridement failed to control the infection, the two-stage revision THA procedure was repeated. All patients underwent weekly follow-up visits for two months, then at three, six and nine months, and biannually thereafter.

A new scoring system for the preoperative evaluation of one- and two-stage revision THAs was developed, based on the six essential variables previously recognised as major risk factors for infection $[6,7,9-12]$. These included (1) patient's general condition, (2) duration of infection and number of prior operations, (3) present wound complications, (4) the presence of microorganisms, (5) CRP levels and (6) the necessity of bone grafting (Fig. 2). Each parameter received 0-2 points, giving a maximum score of 12 points. After the validity of the new scoring system was confirmed, a retrospective analysis was performed by two orthopaedic surgeons blinded to the scoring outcomes.

Prosthesis survival was analysed using the Kaplan-Meier method, with $95 \%$ confidence intervals (CIs); implant removal due to recurrent infection was considered the end point. Univariate Cox proportional hazards models of the association between risk factors and implant removal were built. All independently associated variables were included in the multivariate Cox analysis. In addition, the first episode of recurrent infection was analysed, but further episodes were censored. The validity of the scoring system was evaluated using receiver-operating characteristic (ROC) curves. The ROC analysis was performed using the parameters identified in the multivariate analysis to define the threshold for implant removal, facilitating the calculation of sensitivity and specificity. Prognostic sensitivity was defined as the total number 
Fig. 1 Study flow chart

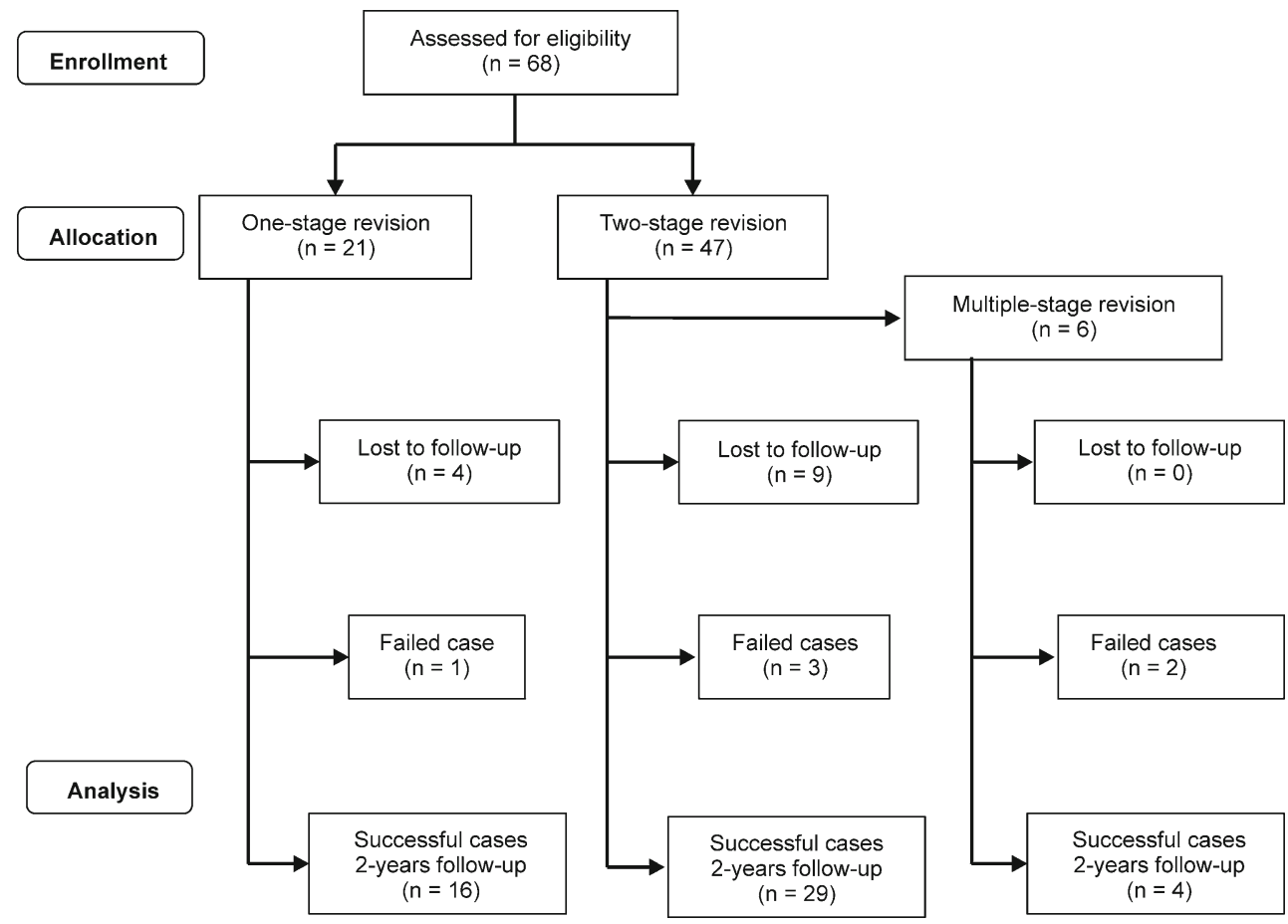

of cases divided by the number of implant removals. The ROC curve was constructed by plotting the sensitivity, on the vertical axis, and 100 minus the specificity, on the horizontal axis, for a given cut-off point. Using ROC analysis, the thresholds for the best sensitivity and specificity of the scoring system were defined, and thereby the most valuable cut-off point for each score was estimated. A two-way table of the scores and outcomes was constructed to predict the risk of recurrence for each score. All data were analysed using oneway analysis of variance (ANOVA), using SAS version 9.2

Criterion

Points

General condition

Poor (American Society of Anesthesiologists' class $\geq 3$, etc.)

Moderate (diabetes mellitus, steroid, autoimmune disease, etc.)

Good

2. Duration of infection, number of past operations

Past operations $\geq 2$ (including osteotomy, debridement, etc.)

Late infection

Early or delayed infection

3. Present wound complication

Sinus tract, abscess

Slightly damaged (reddish, local heat, etc.) Intact

4. Presence of microorganisms

Methicillin-resistant organisms, Gram-negative organisms

Unknown

Antibiotic sensitive organisms

5. C-reactive protein ( $\mathrm{mg} / \mathrm{L})$

$>50$

$5-50$

$<5$

$($ Total $=12)$

6. Necessity for bone grafting

Necessary

Unnecessary

Fig. 2 Pre-operative scoring system
(SAS Institute, Cary, NC, USA); a $p$ value $<0.05$ was considered significant.

\section{Results}

Patient demographic data are shown in Table 1, and the microorganisms isolated pre- and intraoperatively are shown in Table 2. Among patients undergoing one-stage revision THA, 16 of 17 cases were successful, and among patients undergoing two-stage revision THA, including multiple-stage revision THA, 33 of 38 cases were successful (Fig. 3). The ten year survival rate was $94 \%$ (95\% CI 83-100) for one-stage revision THA and $87 \%$ (95\% CI 76-98) for the two-stage revision THA.

Significant univariate risk factors for implant removal due to recurrent infection were general condition [hazard ratio (HR) $0.13 ; 95 \%$ CI $0.03-0.51 ; p=0.003$ ], the presence of microorganisms (HR 0.42; $95 \%$ CI $0.18-1.00$; $p=0.049$ ) and CRP level (HR 0.27; $95 \%$ CI 0.08-0.86; $p=0.027$ ). In the multivariate Cox regression analysis, the total preoperative score was an independent risk factor for implant removal due to recurrent infection (HR 0.17; $95 \%$ CI $0.06-0.49 ; p=0.001$ ). To confirm the validity of the scoring system, the sensitivity and specificity were plotted on an ROC curve (area under the curve 0.97; $95 \%$ CI 0.91-1.00) (Fig. 4a). The sensitivity and specificity, at a scoring system cut-off of 4 points, were 83 and $100 \%$, respectively; the risk of recurrent infection in patients 
Table 1 Patient demographics

\begin{tabular}{lll}
\hline & One-stage revision THA $(n=17)$ & Two-stage revision THA $(n=38)$ \\
\hline Mean age at time of surgery (years) & $69(52-90)$ & $64(34-86)$ \\
Male to female ratio & $5: 12$ & $7: 31$ \\
THA to hemiarthroplasty ratio & $11: 6$ & $21: 17$ \\
Mean time from primary procedure to first-stage revision (years) & $4(0.3-12)$ & $4(0.1-14)$ \\
Mean time from first-stage to second-stage revision (weeks) & $6(0.7-11)$ & $8(5-13)$ \\
Mean time from revised THA to final follow-up (years) & & $5(0.7-10)$ \\
\hline
\end{tabular}

The values show the mean (range)

THA total hip arthroplasty

scoring 4 points was $83 \%$. The risk of infection recurrence is graphically illustrated in Fig. $4 \mathrm{~b}$.

The preoperative scores (Fig. 5) for successful cases were 8-12 points for patients undergoing one-stage revision THA and 5-9 points for those undergoing two-stage revision THA. In addition, for patients undergoing one-stage revision THA, the average total pre-operative scores for successful and failed cases were 9.9 and 6.0, respectively. The average total preoperative scores for patients undergoing successful two-stage revision THAs, multiple-stage revision THAs and failed twostage revision THAs were 7.2, 5.8 and 3.6, respectively. There were significant differences between the mean preoperative scores of patients undergoing successful one- and two-stage revision THAs $(p<0.05)$. Among patients undergoing twostage revision THAs, there were significant differences in the mean preoperative scores between the successful and failed cases $(p<0.05)$.

\section{Discussion}

The current consensus, based on algorithms and staging systems for periprosthetic hip infections [6-10], is that one-stage revision THA can be performed when required by patient circumstances. However, a scoring system is not available to help determine when a one- or two-stage revision THA surgical strategy is appropriate. We selected individual risk factors, based on the published criteria [6, 7, 9-12], and attempted to set up a new scoring system for pre-operative evaluations.

Despite the promise of the novel scoring system described here, there are some limitations to this study. First, the study sample size was small, involving only 55 individuals. Obtaining a large series from a single institution is difficult, and there have been only five literature reports involving relatively large patient series ( $>50$ patients) from a single institution [15-19]. We considered an analysis from a single

Table 2 Microorganisms isolated pre- and intra-operatively

\begin{tabular}{lll}
\hline Isolates & One-stage revision THA & Two-stage revision THA \\
\hline CNS & 4 & 10 \\
MRSA & 2 & 8 \\
MSSA & 1 & 6 \\
MSSE & 3 & 2 \\
MRSE & 1 & 1 \\
Pseudomonas aeruginosa & 0 & 2 \\
Staphylococcus epidermidis & 1 & 1 \\
MRCNS & 0 & 1 \\
Streptococcus anginosus & 0 & 1 \\
Serratia sp. & 0 & 1 \\
Micrococcus sp. & 1 & 0 \\
Enterococcus sp. & 0 & 1 \\
Peptostreptococcus sp. & 0 & 1 \\
Unknown & 6 & 5 \\
\hline
\end{tabular}

THA total hip arthroplasty, CNS coagulase-negative Staphylococcus, MRSA methicillin-resistant Staphylococcus aureus, MSSA methicillin-sensitive Staphylococcus aureus, MSSE methicillin-sensitive Staphylococcus epidermidis, MRSE methicillin-resistant Staphylococcus epidermidis, MRCNS methicillin-resistant coagulase-negative Staphylococcus 
Fig. 3 Radiographs of a 75-yearold woman who underwent twostage revision THA (scoring system, 7 points). a Combined deficiencies, acetabular defect. $\mathbf{b}$ ALAC beads seven weeks after the first-stage surgery. c Five years after reimplantation with allograft reconstruction and a metal ring
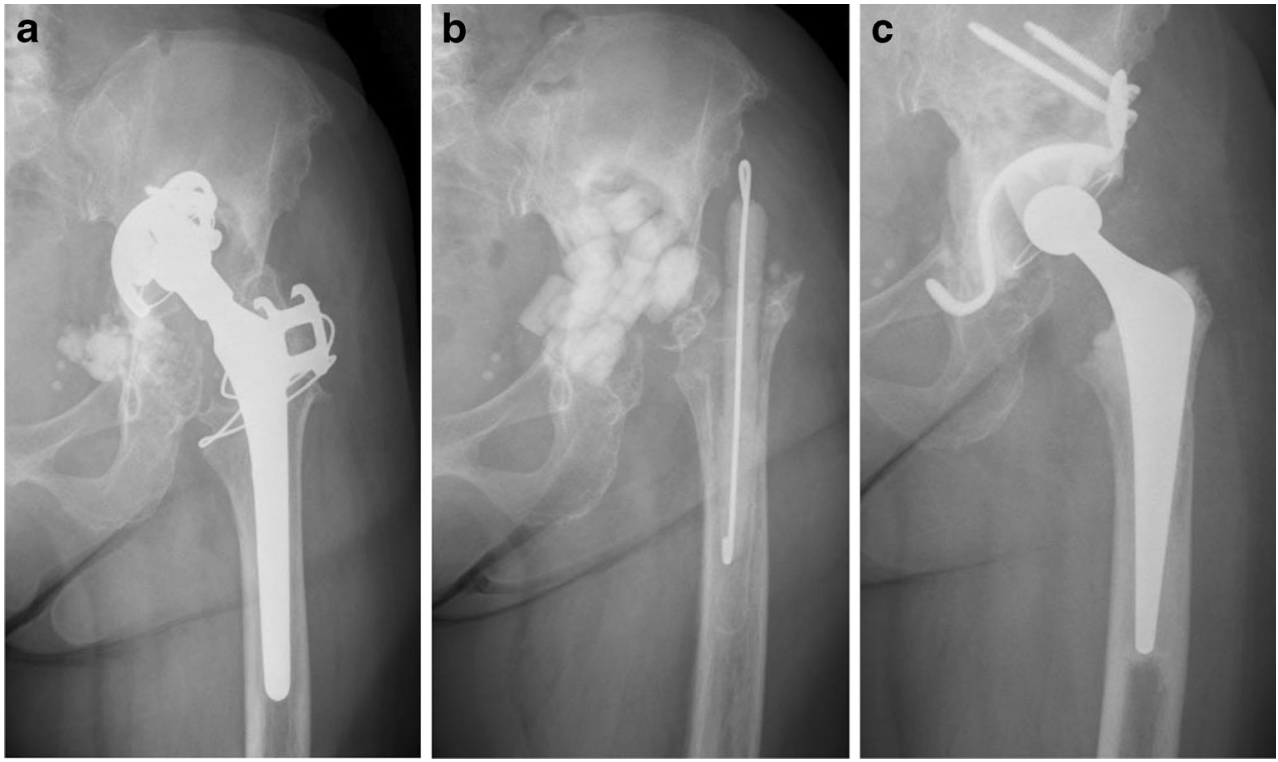

institution more valuable as studies included in meta-analyses might include slightly differing techniques performed by surgeons. Second, although the validity of the new scoring system was confirmed (Fig. 4a), confirmation of the veracity of each established parameter was not possible. For example, Wroblewski [12], Elson [15] and Hsieh et al. [20] recommended two-stage revision THAs in cases where bone grafting was necessary, whereas Rudelli et al. [21] reported that among 32 patients, including those with sinus tracts, the recurrence rate was $94 \%$ for one-stage revision THA. Among patients with sinus tracts, Raut et al. [22] and Ure et al. [23] reported that infection was controlled in 90 and $100 \%$ of patients, respectively. Furthermore, in our study, we did not explore other potential variables such as implant loosening, polymicrobial infection and haematogenous infection for the scoring system. Third, some patients, not undergoing implant removal, may have actually had recurrent infections.
However, if these cases were not diagnosed as being infected, according to the working definition, they were not considered recurrent infections. Determination of the necessity of surgical treatment is important, and we defined a failure as an implant removal due to recurrent infection. In addition, the duration of antibiotic therapy after reimplantation during a two-stage revision THA may not be universally consistent. Zimmerli et al. [24] suggested that, in two-stage revision THAs, if intraoperative specimen cultures remain negative, antimicrobial treatment may be discontinued after six weeks; otherwise, therapy should continue for three months. However, our duration was not congruent with international benchmarks.

Jackson and Schmalzried [11] conducted an infection control analysis of 1,299 cases of THA from 12 studies to determine the factors associated with successful patient outcomes. They suggested that cementless implants may be contraindicated in patients requiring one-stage revision THAs. In our
Fig. 4 a ROC curves. b Percentage risk of recurrence for each score
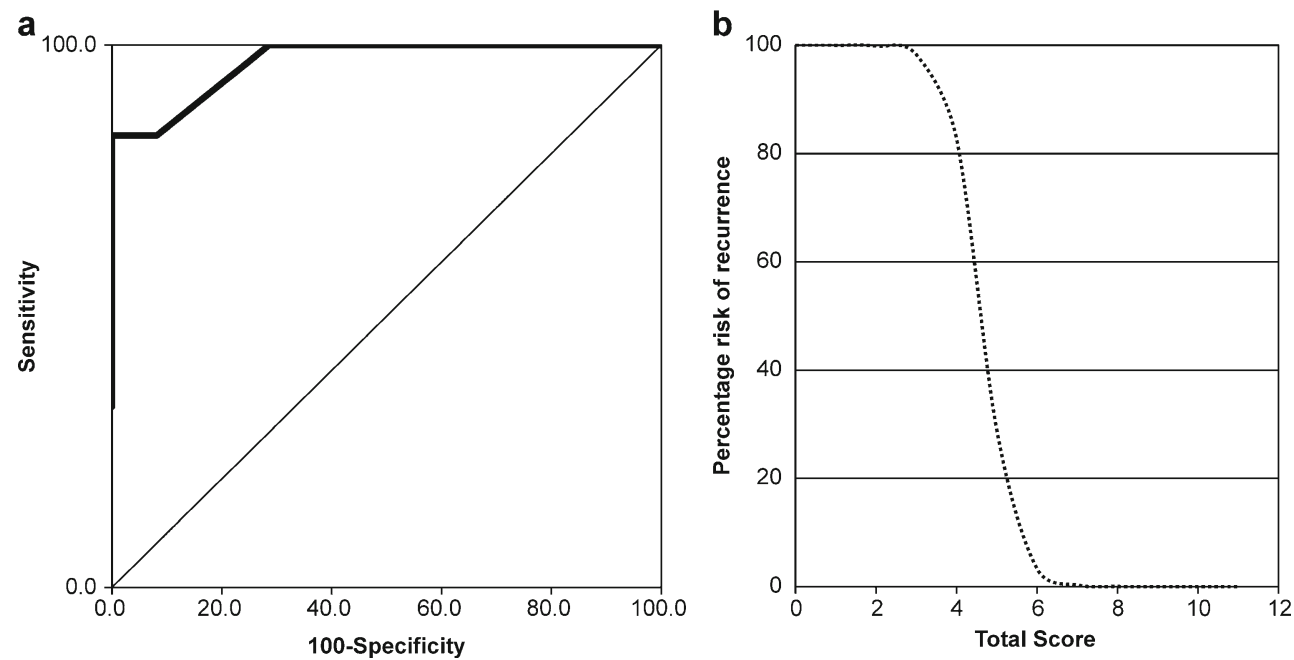
Fig. 5 Total preoperative scores for patients undergoing one- and two-stage THA

(points)

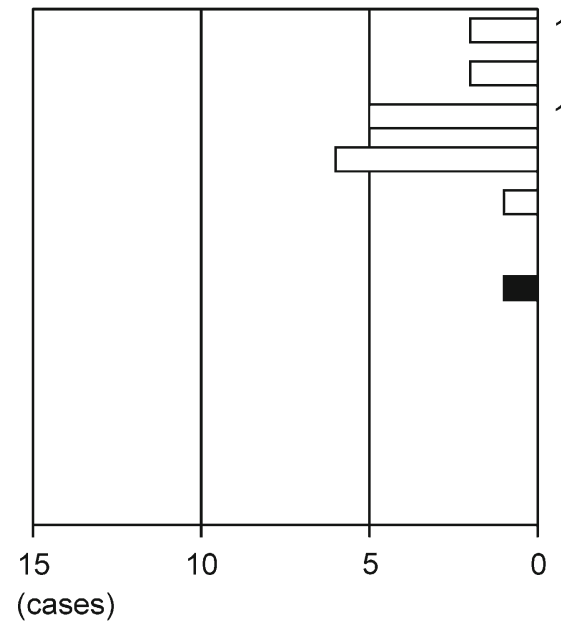

One-stage revision THA

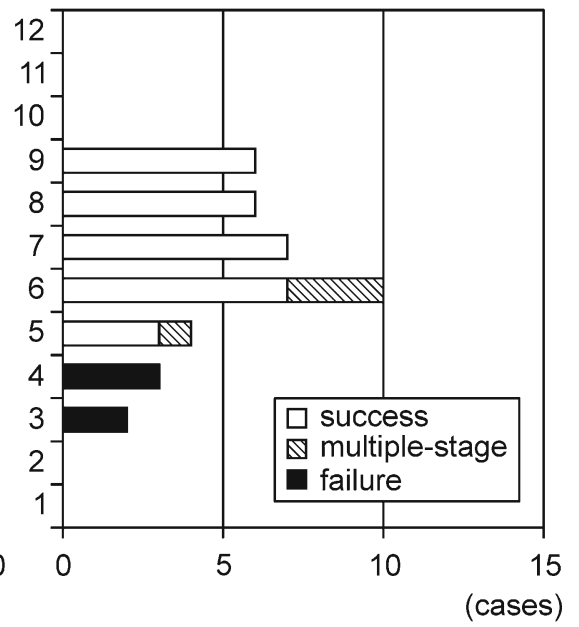

Two-stage revision THA study, the ten year survival rates were 94 and $87 \%$ for oneand two-stage revision THAs, respectively, although selection bias may undermine the validity of this direct comparison. Consequently, one-stage revision THAs involving ALAC gave promising results because of their advantages for the treatment and prevention of infection. According to previous reports (Table 3), some surgeons routinely perform one-stage revision THAs, with infection control rates in six studies reported to be $77-100 \%[1,2,12,22,23,25]$. Others, including our study, selected one-stage revision THA depending on patient requirements and showed infection control rates of $76-100 \%$ in the eight studies $[13,15-19,26]$. These rates were similar to those reported in studies only performing onestage revision THAs. The indications for one-stage revision THAs are shown in Table 3, but there are few reports describing the use of definite algorithms [14, 19]. Oussedik et al. [18] suggested that a direct comparison between one- and twostage revision THA is difficult, given the non-randomised nature of such studies, but that including those patients treated by two-stage revision THA can provide an estimate of the proportion of cases that might be suitable for one-stage revision THA. According to the pre-operative scoring system, there were significant differences between the scores corresponding to successful one- and two-stage revision THAs;

Table 3 Recently published studies reporting relatively large series of one-stage revision THAs from a single institution

\begin{tabular}{|c|c|c|c|c|c|c|}
\hline \multirow[t]{2}{*}{ First author } & \multirow[t]{2}{*}{ Year published } & \multicolumn{2}{|c|}{ One-stage revision THA } & \multicolumn{2}{|c|}{ Two-stage revision THA } & \multirow[t]{2}{*}{ Indication } \\
\hline & & $n$ & Infection control (\%) & $n$ & Infection control (\%) & \\
\hline Buchholz [13] & 1981 & 667 & 77 & - & & No selection \\
\hline Miley [25] & 1982 & 47 & 87 & - & & No selection \\
\hline Wroblewski [12] & 1986 & 102 & 91 & - & & No selection \\
\hline Raut [22] & 1994 & 57 & 86 & - & & No selection \\
\hline Ure [23] & 1998 & 20 & 100 & - & & No selection \\
\hline Callaghan [2] & 1999 & 24 & 92 & - & & No selection \\
\hline Sanzén [19] & 1988 & 72 & 76 & 30 & 75 & $\mathrm{GC}, \mathrm{BG}$ \\
\hline Hope [16] & 1989 & 72 & 88 & 19 & 100 & $\mathrm{PO}, \mathrm{MO}, \mathrm{BG}$ \\
\hline Elson [15] & 1993 & 235 & 88 & 61 & 97 & Not described \\
\hline Garvin [26] & 1994 & 10 & 90 & 30 & 97 & DI, WC, MO \\
\hline Klouche [17] & 2000 & 38 & 100 & 46 & 98 & DI, MO, BG \\
\hline Giulieri [13] & 2004 & 16 & 94 & 31 & 90 & Zimmerli’s algorithm [10] \\
\hline Oussedik [18] & 2010 & 11 & 100 & 39 & 95 & Haddad's protocol [6] \\
\hline Our study & 2014 & 17 & 94 & 38 & 87 & GC, DI, PO, WC, MO, CRP, BC \\
\hline
\end{tabular}

$T H A$ total hip arthroplasty, $G C$ general condition, $B G$ bone grafting, $P O$ past operations, $M O$ microorganisms, $D I$ duration of infection, $W C$ wound complication, $C R P$ C-reactive protein 
one-stage revision THA was recommended in cases scoring more than 9 points (Fig. 5). In contrast, the risk of recurrent infection in patients scoring 4 points was $83 \%$ (Fig. 4).

In conclusion, the novel preoperative scoring system described here was useful for determining the management of periprosthetic hip infections. One-stage revision THA is recommended for patients scoring more than 9 points, and other options should be considered for patients scoring 4 or fewer points in order to minimise the risk of recurrent infection. Additionally, one-stage revision THAs may be possible for cases scoring 7 or 8 points. To fully validate this novel scoring system, a prospective study of the system in a multicentre trial is required.

Conflict of interest The authors declare that they have no conflict of interest.

Open Access This article is distributed under the terms of the Creative Commons Attribution License which permits any use, distribution, and reproduction in any medium, provided the original author(s) and the source are credited.

\section{References}

1. Buchholz HW, Elson RA, Engelbrecht E, Lodenkämper H, Röttger J, Siegel A (1981) Management of deep infection of total hip replacement. J Bone Joint Surg Br 63:342-353

2. Callaghan JJ, Katz RP, Johnston RC (1999) One-stage revision surgery of the infected hip. A minimum 10-year followup study. Clin Orthop Relat Res 369:139-143

3. Garvin KL, Hanssen AD (1995) Infection after total hip arthroplasty. Past, present, and future. J Bone Joint Surg Am 77:1576-1588

4. Lange J, Troelsen A, Thomsen RW, Søballe K (2012) Chronic infections in hip arthroplasties: comparing risk of reinfection following one-stage and two-stage revision: a systematic review and metaanalysis. Clin Epidemiol 4:57-73

5. Langlais F (2003) Can we improve the results of revision arthroplasty for infected total hip replacement? J Bone Joint Surg Br 85:637-640

6. Haddad FS, Masri BA, Garbuz DS, Duncan CP (1999) The treatment of the infected hip replacement. The complex case. Clin Orthop Relat Res 369:144-156

7. Hanssen AD, Osmon DR (2000) Assessment of patient selection criteria for treatment of the infected hip arthroplasty. Clin Orthop Relat Res 381:91-100

8. McPherson EJ, Woodson C, Holtom P, Roidis N, Shufelt C, Patzakis $M$ (2002) Periprosthetic total hip infection: outcomes using a staging system. Clin Orthop Relat Res 403:8-15

9. Parvizi J, Adeli B, Zmistowski B, Restrepo C, Greenwald AS (2012) Management of periprosthetic joint infection: the current knowledge: AAOS exhibit selection. J Bone Joint Surg Am 94:e104
10. Zimmerli W, Ochsner PE (2003) Management of infection associated with prosthetic joints. Infection 31:99-108

11. Jackson WO, Schmalzried TP (2000) Limited role of direct exchange arthroplasty in the treatment of infected total hip replacements. Clin Orthop Relat Res 381:101-105

12. Wroblewski BM (1986) One-stage revision of infected cemented total hip arthroplasty. Clin Orthop Relat Res 211: $103-107$

13. Giulieri SG, Graber P, Ochsner PE, Zimmerli W (2004) Management of infection associated with total hip arthroplasty according to a treatment algorithm. Infection 32:222-228

14. Della Valle C, Parvizi J, Bauer TW, Dicesare PE, Evans RP, Segreti J, Spangehl M, Watters WC, Keith M, Turkelson CM, Wies JL, Sluka P, Hitchcock K, American Academy of Orthopaedic Surgeons (2010) Diagnosis of periprosthetic joint infections of the hip and knee. J Am Acad Orthop Surg 18:760-770

15. Elson RA (1993) Exchange arthroplasty for infection. Perspectives from the United Kingdom. Orthop Clin North Am 24:761-767

16. Hope PG, Kristinsson KG, Norman P, Elson RA (1989) Deep infection of cemented total hip arthroplasties caused by coagulase-negative staphylococci. J Bone Joint Surg Br 71: $851-855$

17. Klouche S, Leonard P, Zeller V, Lhotellier L, Graff W, Leclerc P, Mamoudy P, Sariali E (2012) Infected total hip arthroplasty revision: one- or two-stage procedure? Orthop Traumatol Surg Res 98:144 150

18. Oussedik SI, Dodd MB, Haddad FS (2010) Outcomes of revision total hip replacement for infection after grading according to a standard protocol. J Bone Joint Surg Br 92:1222-1226

19. Sanzén L, Carlsson AS, Josefsson G, Lindberg LT (1988) Revision operations on infected total hip arthroplasties. Twoto nine-year follow-up study. Clin Orthop Relat Res 229:165172

20. Hsieh PH, Shih CH, Chang YH, Lee MS, Yang WE, Shih HN (2005) Treatment of deep infection of the hip associated with massive bone loss: two-stage revision with an antibiotic-loaded interim cement prosthesis followed by reconstruction with allograft. J Bone Joint Surg Br 87:770-775

21. Rudelli S, Uip D, Honda E, Lima AL (2008) One-stage revision of infected total hip arthroplasty with bone graft. J Arthroplasty 23: $1165-1177$

22. Raut VV, Siney PD, Wroblewski BM (1994) One-stage revision of infected total hip replacements with discharging sinuses. J Bone Joint Surg Br 76:721-724

23. Ure KJ, Amstutz HC, Nasser S, Schmalzried TP (1998) Directexchange arthroplasty for the treatment of infection after total hip replacement. An average ten-year follow-up. J Bone Joint Surg Am 80:961-968

24. Zimmerli W, Trampuz A, Ochsner PE (2004) Prosthetic-joint infections. N Engl J Med 351:1645-1654

25. Miley GB, Scheller AD Jr, Turner RH (1982) Medical and surgical treatment of the septic hip with one-stage revision arthroplasty. Clin Orthop Relat Res 170:76-82

26. Garvin KL, Evans BG, Salvati EA, Brause BD (1994) Palacos gentamicin for the treatment of deep periprosthetic hip infections. Clin Orthop Relat Res 298:97-105 